\title{
Cardioplegia retrógrada seqüencial
}

\author{
Otoni M. GOMES*, Márcio PITCHON*, José M. G. BRUM*, Eros Silva GOMES*, \\ Wagner C. PÁDUA FILHO*, Robert Einstein A. FREITAS*, Marcilio FARAJ*, Wilson de Souza LIMA*
}

RBCCV 44205-311

Gomes O M, Pitchon M, Brum J M G, Gomes E S, Pádua Filho W C, Freitas R E A, Faraj M, Lima W S . Cardioplegia retrógrada seqūencial. Rev Bras Cir Cardiovasc 1996; 11 (3): 208-15.

RESUMO: Estudou-se o padrăo de distribuição miocárdica de solução cardioplégica (SC) cristalóide gelada $\left(3^{\circ} \mathrm{C}=4^{\circ} \mathrm{C}\right)$ perfundida nos coraçôes de 15 câes mestiços com pesos variáveis entre $10-15 \mathrm{~kg}$. Após anestesia e toracotomia mediana anterior, o pericárdio foi aberto, sendo estabelecida circulaçāo extracorpórea. As seguintes vias foram empregadas para injeçāo cardioplégica: 1) Anterógrada - por canulaçāo da aorta ascendente a montante da pinça de oclusăo; 2) Retrógrada Seletiva - através de cánula com balão autoinflável introduzido no seio coronário ( $\mathrm{SCO}$ ); 3) Retrógrada Total - através de cânula introduzida no átrio direito $(A D)$; 4) Retrógrada Sequeencial SCo-AD - com a SC injetada primeiro pelo seio coronário até a temperatura do septo interventricular atingir $16^{\circ} \mathrm{C} \mathrm{e}$, em sequéência, pela cânula no átrio direito como na técnica retrógrada total, com o tronco arterial pulmonar ocluido; 5)Retrógrada Seqüencial SCo-VD-com a cavidade do ventrículo direito perfundida por cânula passada através da valva tricúspide. Controlou-se a variação da temperatura miocárdica no ventrículo esquerdo, VD, AD e região do nó sinoatrial, por meio de teletermómetro Ómega com termistor de agulha. Pode-se constatar que o esfriamento cardiaco uniforme, o menor volume e o menor tempo de injeção ocorreram com a técnica anterógrada, seguida em excelência pelas técnicas retrógradas seqūenciais SCo-AD e SCo-VD. Concluiu-se que a técnica de cardioplegia retrógrada seqủencial é significantemente melhor que as retrógradas seletivas pelo $S C o$ e total pelo $A D$, como usualmente empregadas para proteção miocárdica, quando comparadas com a técnica de perfusăo anterógrada pela aorta.

DESCRITORES: Parada cardiaca, induzida, métodos. Miocárdio, metabolismo. Cardioplegia. Proteçăo miocárdica, cardioplegia.

\section{INTRODUÇĀO}

MELROSE et al. (1), em 1975, introduziram pioneiramente a técnica de cardioplegia, injetando citrato de potássio na aorta ascendente para obter parada cardiaca intra-operatória.

A formulaçăo das soluçōes cardioplégicas sofreu modificaçōes consideráveis a partir dos estudos de REIDEMEISTER ot al. (2), BRETSCHNEIDER et al. (3) e KIRSCH et al. (4), procurando diminuir a excitabilidade muscular pela reduçăo do sódio extracelular, injeção de procaína e aumento de concentração de magnésio. A técnica de injeçăo anterógrada pela canulação da aorta ascendente, entretanto, persiste em uso consagrado, substituída pela canulação direta de cada óstio arterial coronário, como pioneiramente proposto por KAY et al. (5), apenas em operaçōes com abertura da aorta ascendente.

LILLEHEI et al. (6), em 1956, empregaram a perfusăo coronária retrógrada pelo seio coronário,

Trabalho realiza do no Serviço do Corạ̧āo do Hospital Săo Francisco de Assis - Fundação Cardiovascular São Francisco de Assis. Belo Horizonte, MG, Brasil.

Recebido para publicação em junho de 1996.

- Do Serviço do Coração do Hospital Săo Francisco de Assis.

Endereço para correspondência: Otoni Moreira Gomes. Rua Jacui, 1191. Concórdia. Belo Horizonte, MG, Brasil. CEP:31110-050. Telefax: (031) 442-7488. 
em operação de correçăo da estenose valvar aórtica calcificada.

MORAIS ${ }^{(7)}$ e JAZBIK ${ }^{(8)}$, em 1970, estudaram clínica e experimentalmente, pela primeira vez, a perfusão coronária retrógrada total, perfundindo nāo só o seio coronário, mas os vasos de Tebésius e os sinusóides miocárdicos.

Embora o uso da perfusăo sangüinea retrógrada, continua ou intermitente, tenha sido abandonada, face aos bons resultados da cardioplegia por via anterógrada, MENASCHÉ et al. (9) estudaram e demonstraram a eficiência da cardioplegia perfundida por meio de cateter inroduzido no seio coronário, utilizando-a como única via de perfusăo cardioplégica (10).

Sabe-se, contudo, que a perfusăo coronária retrógrada não percorre a mesma via de perfusāo capilar que a anterógrada, fisiológica.

DRINKWATER et al. (11) e PARTINGTON et al. (12) propuseram a associação da perfusão cardioplégica anterógrada com a retrógrada pelo seio coronário, para que fossem protegidas regiōes miocárdicas não alcançadas com o uso isolado de cada uma das técnicas, principalmente em pacientes com obstruçōes coronárias múltiplas.

Esta proposiçāo justifica-se frente à demonstraçāo por STIRLING et al. (13), estudando perfusăo cardioplégica com microesferas radioativas, de que a injeção retrógrada pelo seio coronário năo perfunde uniformemente o coração, deixando desprotegidas a porçăo média do ventrículo direito e a região póstero-septal do ventrículo esquerdo.

Considerando as situações clínicas em que a perfusăo coronária anterógrada, estando severamente impedida, năo pode suprir as falhas da perfusão retrógrada, conforme atualmente empregada, desenvolveu-se a presente investigação. Seu objetivo é estudar experimentalmente, em cães, as vantagens do emprego de método que associa perfusăo seqũencial pelo seio coronário e pelo átrio direito. ou pelo seio coronário e diretamente pelo ventrículo direito: a extensão da proteção miocárdica obtida com a técnica ora proposta de cardioplegia retrógrada sequencial é comparada com os resultados alcançados com as técnicas anterógradas, retrógrada seletiva pelo seio coronário e retrógrada total pelo átrio direito, em função dos gradientes térmicos mapeados em diferentes zonas cardíacas, do tempo necessário de infusăo cardioplégica e do volume necessário em cada técnica.

\section{MATERIAL E MÉTODOS}

Foram estudados 15 cães mestiços, com pesos variáveis entre $10 \mathrm{~kg}$ e $15 \mathrm{~kg}$ de peso corpóreo, submetidos a anestesia geral com Thiopental Sódico e ventilação controlada com respiração artificial.

Após toracotomia mediana longitudinal, o pericárdio foi aberto no mesmo sentido, expondo o coração e vasos da base, que foram dissecados $\theta$ contornados por fita de algodão.

Foi estabelecida circulação extracorpórea hipotérmica $\left(30^{\circ} \mathrm{C}\right)$ com derivaçāo total do retorno venoso-dupla canulaçăo de cavas - e perfusão pela artéria femoral comum esquerda.

Em todos os casos, a descompressão das câmaras esquerdas foi realizada por abertura da aurícula esquerda.

A veia ázigo foi ligada em todos os animais e as veias cavas garroteadas antes do ínicio da perfusão cardioplégica.

Uma cânula foi introduzida na porção ascendente da aorta, à montante da pinça de sua oclusão.

Essa cânula foi utilizada para injeçāo de solução cardioplégica na técnica anterógrada e para aspiraçäo nas técnicas retrógradas. Uma segunda cânula, introduzida na mesma regiāo aórtica, foi unida a manômetro de mercúrio para controle da pressão de perfusão coronária arterial, mantida em $70 \mathrm{mmHg}$.

Para perfusăo cardioplégica retrógrada seletiva delimitou-se na parede atrial direita uma àrea de aproximadamente $1 \mathrm{~cm}$ de diâmetro, circundada por sutura em bolsa com fio de Polipropileno 4.0; através desta zona, introduziu-se cânula com balonete auto-inflável (Research Medical Inc., Salt Lake City, USA), que foi dirigida e impactada no seio coronário.

Para perfusão cardioplégica retrógrada total, através de zona delimitada por sutura, introduziu-se no átrio direito cânula plástica, $\operatorname{com} 3 \mathrm{~mm}$ de diâ. metro.

Nestes casos o tronco pulmonar foi ocluido durante todo o período de injeçăo cardioplégica.

Antes do ínicio da perfusão cardioplégica em cada uma das técnicas estudadas, estabilizou-se a temperatura do septo interventricular (terço médio) em $30^{\circ} \mathrm{C}$, empregando-se o sistema de permutaçăo térmica do circuito extracorpóreo composto com oxigenador de bolhas descartável (FLVMEN - Produtos Médicos Ltda, Volta Redonda - RJ).

Em 9 animais, este mesmo sistema possibilitou - reaquecimento cardiaco, após descompressão aórtica para aproveitamento no estudo subsequeente de outra via de perfusão cardioplégica.

A soluçāo cardioplégica foi injetada com temperatura de $4,0^{\circ} \mathrm{C}$, tendo a seguinte composiçăo: soluçāo de Ringer, $450 \mathrm{ml}$; cloreto de potássio a $10 \%$, 
Gomes O M, Pitchon M, Brum J M G, Gomes E S, Pádua Filho W C, Freitas R E A, Faraj M, Lima W S - Cardioplegia retrógrada sequencial. Rev Bras Cir Cardiovasc 1996;11 (3): 208-15.

TABELA 1

VARIAÇĀO DA TEMPERATURA CARDIACA $\left({ }^{\circ} \mathrm{C}\right)$ - RESULTADOS EM MÉDIAS

\begin{tabular}{|c|c|c|c|c|c|c|c|c|c|c|}
\hline \multirow[b]{2}{*}{$\begin{array}{l}\text { CARDIOPLEGIA } \\
\text { TECNICA }\end{array}$} & \multirow[b]{2}{*}{$\underset{N^{0}}{E X P}$} & \multirow[b]{2}{*}{$\begin{array}{l}\text { SEPTO } \\
\text { MÉDIO }\end{array}$} & \multicolumn{3}{|c|}{ VE } & \multicolumn{3}{|c|}{ VD } & \multirow[b]{2}{*}{$\begin{array}{c}\text { ÁTRIO } \\
\text { DIREITO }\end{array}$} & \multirow[b]{2}{*}{$\begin{array}{l}\text { REGIĀO } \\
\text { SINUSAL }\end{array}$} \\
\hline & & & PONTA & MEIO & BASE & MARGEM & MEIO & SAIDA & & \\
\hline Anterógrada & 6 & 16,0 & 19,2 & 17,3 & 17,1 & 16,8 & 16,8 & 17,0 & 18,7 & 18,6 \\
\hline Retrógrada SCo & 6 & 16.0 & 18,3 & 17,4 & 18,4 & 19,5 & 18.3 & 19,0 & 19,3 & 20,0 \\
\hline Retrógrada Atrial & 6 & 16,0 & 23,0 & 22,0 & 20,5 & 14,5 & 14,2 & 13,5 & 13,5 & 14,5 \\
\hline Retrógrada SCO.AD & 6 & 16.0 & 18,4 & 17,7 & 18.7 & 14,4 & 14.5 & 14,3 & 13,3 & 13,7 \\
\hline Retrógrada SCo-VD & 6 & 16,0 & 18,6 & 17,6 & 18,3 & 13,8 & 14,3 & 14,3 & 19,4 & 20,1 \\
\hline
\end{tabular}

$10 \mathrm{ml}$; bicarbonato de sódio $8,4 \%, 2 \mathrm{ml}$; glicose a $50 \%, 10 \mathrm{ml}$ e xilocaína $1 \%, 2 \mathrm{ml}$.

Em todos os casos a perfusăo com solução cristalóide foi mantida até que a temperatura do septo interventricular (terço médio, anterior) descesse para $16^{\circ} \mathrm{C}$.

Na cardioplegia retrógrada seqūencial em 6 experimentos a perfusāo foi iniciada pela cânula do seio coronário, até o esfriamento septal pré-estabelecido, seguindo-se a perfusāo pela cânula atrial, até o esfriamento da porção média do ventrículo direito descer abaixo de $15^{\circ} \mathrm{C}$. Em outros 6 experimentos, após a perfusāo do seio coronário, a injeçāo continuou por uma cânula posicionada dentro da cavidade ventricular direita.

A pressão de perfusāo variou de $70 \mathrm{mmHg}$ na injeção anterógrada para $20 \mathrm{mmHg}$ na retrógrada seletiva e $40 \mathrm{mmHg}$ na retrógrada total.

Para aferição da temperatura miocárdica empregou-se termômetro digital com termístor de agutha marca Ómega INC. - USA.

A temperatura foi aferida inicialmente no terço intermédio do septo interventricular (posição 1) e posteriormente nas seguintes posiçōes: 2) ponta do ventriculo esquerdo (VE); 3) $1 / 3$ médio da borda do VE; 4) base do VE; 5) margem do ventriculo direito (VD); 6) terço médio do VD; 7) via de saída do VD (subpulmonar); 8) parte média do átrio direito; 9) regiăo sinusal, ou da junçăo cava superior - AD.

Foram registrados os valores térmicos, o volu- me de soluçăo infundida, o tempo de infusăo e a pressão de perfusăo cardioplégica.

Os resultados obtidos foram submetidos a tratamento estatístico (teste " $\mathrm{T}$ " de Student).

\section{RESULTADOS}

A Tabela 1 apresenta os resultados, em médias, das variaçōes de temperatura nas diferentes àreas do miocárdio após perfusão cardioplégica.

Pode-se constatar que, embora a temperatura septal tenha sido semelhante $\left(16^{\circ} \mathrm{C}\right)$, os resultados da cardioplegia anterógrada foram mais uniformes quanto ao esfriamento de VE, VD e AD. Na cardioplegia retrógrada pelo seio coronário nota-se que a perfusão do VD foi menos eficaz, mantendo níveis térmicos mais altos $(p<0,05)$. Na técnica retrógrada total, os niveis térmicos săo mais altos no VE do que nas anteriores $(p<0,05)$; por outro lado, notase maior proteção de VD e AD, com médias de temperatura mais baixas $(p<0,01)$. Com a cardioplegia retrógrada seqüencial, os niveis térmicos atingidos não são estatisticamente diferentes daqueles encontrados no VE com a técnica anterógrada ( $p>0,01)$, nem os valores de VD são diferentes ( $p>0,01$ ) daqueles da cardioplegia retrógrada total. Nota-se, entretanto, que entre as duas técnicas de cardioplegia retrógrada seqūencial a proteção do $A D$ e da região do nó sinoatrial foi maior na variante SCo-AD que na SCo-VD $(p<0,01)$.

A Tabela 2 descreve resultados em médias, das

TABELA 2

DADOS TÉCNICOS - RESULTADOS EM MÉDIAS

\begin{tabular}{lccccc}
\hline $\begin{array}{l}\text { CARDIOPLEGIA } \\
\text { TÉCNICA }\end{array}$ & $\begin{array}{c}\text { EXP } \\
\mathrm{N}^{9}\end{array}$ & $\begin{array}{c}\text { PRESSĀO } \\
(\mathbf{m m H g})\end{array}$ & $\begin{array}{c}\text { DURAÇĀO } \\
(\mathbf{m i n})\end{array}$ & $\begin{array}{c}\text { VOLUME } \\
(\mathbf{m l})\end{array}$ & EFEITO ADVERSO \\
\hline Anterógrada & 6 & 70 & 2,18 & 167 & - \\
Retrógrada SCo & 6 & 20 & 4,04 & 442 & \\
Retrógrada Total & 6 & 40 & 8,01 & 828 & Distensão(AD-VD) \\
Retrógrada SCo-AD & 6 & $20-40$ & 4,32 & 503 & Distensão(AD-VD) \\
Retrógrada SCo-VD & 6 & $20-40$ & 4,25 & 453 & Distensão (VD) \\
\hline
\end{tabular}


Gomes O M, Pitchon M, Brum J M G, Gomes E S, Pádua Filho W C. Freitas R E A, Faraj M, Lima W S - Cardioplegia retrógrada sequencial. Rev Bras Cir Cardiovasc 1996;11 (3): 208-15.

\section{VARIAÇÄO DA TEMPERATURA MIOCÁADICA - MEIO DE VENTRICULO ESQUERDO}

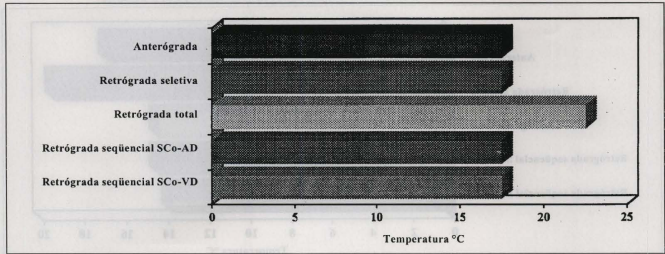

variações pressóricas, do volume injetado e do tempo de infusão em cada uma das técnicas utilizadas.

Por via anterógrada injetou-se volume de 167 ml durante 2,18 minutos, em média; seletivamente, pelo seio coronário; esses valores aumentaram para $442 \mathrm{ml}(p<0,01)$ e 4,04 minutos $(p<0,01)$; na cardioplegia retrógrada total, subiram para $828 \mathrm{ml}(p<$ $0,01)$ e 8,01 minutos $(p<0,01)$, respectivamente. Com as cardioplegias retrógradas sequenciais os volumes empregados foram $503 \mathrm{ml}$ e $453 \mathrm{ml}$, 。 tempo de perfusăo igual a 4,32 e 4,25 minutos, respectivamente, para as técnicas SCo-AD e SCoVD. Embora estes valores sejam estatisticamente significantes quando comparados com os da cardio- plegia anterógrada $(p<0,01)$, năo diferem $(p>0,01)$ daqueles obtidos com a cardioplegia retrógrada seletiva isoladamente. Os Gráficos 1 a 4 detalham os resultados mais importantes.

\section{COMENTÁRIOS}

Historicamente, as seguintes vias de perfusăo coronária artificial foram propostas e estudadas: perfusāo anterógrada coronária, a partir da perfusāo arterial sistêmica, retrógrada, pela artéria femorai, ou da perfusão sistêmica, por cânula introduzida na aorta ascendente (14). No Brasii, este método foi

GRÁFICO 2

VARIAÇĀO DA TEMPERATURA MIOCÁRDICA - BASE DO VENTRICULO ESQUERDO

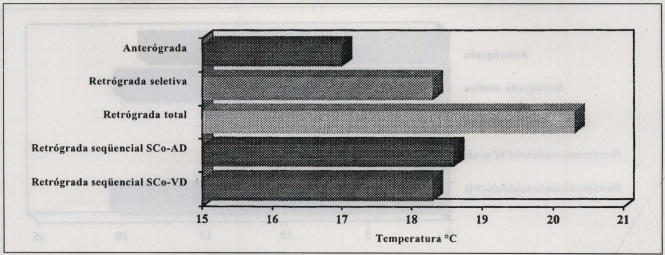


Gomes O M, Pitchon M, Brum J M G, Gomes E S, Pádua Filho W C, Freitas R E A, Faraj M, Lima W S - Cardioplegia retrógrada sequencial. Rev Bras Cir Cardiovasc 1996;11 (3): 208-15.

\section{GRÁFICO 3}

VARIAÇÃO DA TEMPERATURA MIOCARDICA - MARGEM DO VENTRICULO DIREITO

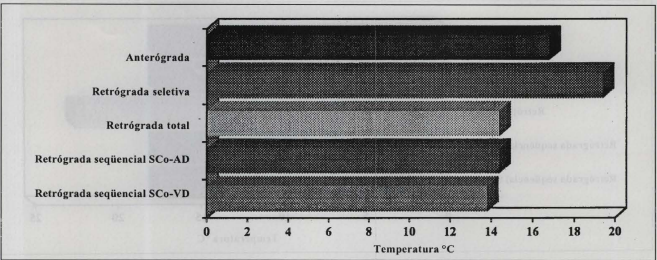

pioneiramente sistematizado por MORAIS et al. (15); perfusāo antegrógrada por canulaçāo direta dos óstios coronários (KAY et al., 1958 (5); perfusāo retrógrada seletiva pelo seio coronário (LILLEHEI et al., $\left.1956{ }^{(6)}\right)$; perfusāo retrógrada total, atrial (MORAIS, $1969^{(7)}$; JAZBIK et al., $1969^{(16)}$ e JAZBIK, $\left.1970^{(8)}\right)$.

A despeito da eficácia de todas estas vias para manutençăo da viabilidade cardiaca, seu uso para manutenção de perfusăo coronária continua foi abandonado face à exigência de aumento na manipulaçăo operatória, desconforto técnico e pelo risco de lesão vascular coronária, macro ou microscópica (lesão de óstio coronário, edema, rotura do seio coronário, bloqueio atrioventricular, distensão e dissociaçāo de miofibrilas).

O advento e confirmaçăo universal da eficácia da cardioplegia trouxe, após período de efervescência na discussão de qual a melhor formulaçăo. a preocupação quanto à melhor técnica para perfusảo mais homogênea do miocárdio. As seguintes opções tèm sido questionadas: cardioplegia anterógrada, por injeçăo na aorta ascendente, como iniciada por MELROSE et al. (1), em 1955, ou diretamente nos óstios coronários. BRAILE et al. (17) utilizaram estas vias, nos estudos pioneiros da cardioplegia em nosso pais; Cardioplegia hipotérmica tópica, como proposta por SHUMWAY et al., em

GRÁFICO 4

VARIAÇĀO DA TEMPERATURA MIOCÁRDICA - REGIĀO DO NÓ SINOATRIAL

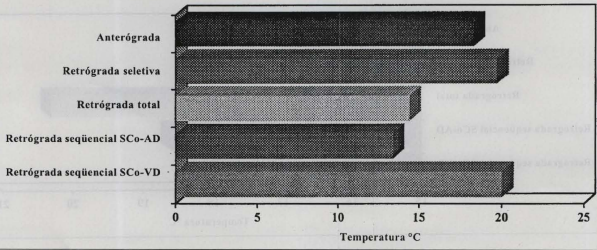


$1959{ }^{(18)}$, e utilizada com modificaçōes por HUFNAGEL et al., em 1961 (19); e JATENE (1963) (20), entre nós; cardioplegia retrógrada seletiva pelo seio coronário (MENASCHÉ et al., $1982(9)$; cardioplegia retrógrada total, atrial (FABIANI et al., 1986 (21))

O interesse pela cardioplegia retrógrada progride na medida em que se definem as falhas da perfusão anterógrada, quer por motivos técnicos, como em operações na aorta ascendente - com destaque para a estenose de óstio coronário (calcificação, anomalia congênita, ateroma) - ou na obstrução coronária.

Fator especial constitui a hipertrofia miocárdica, mesmo na vigência de coronárias normais, onde a relaçăo àrea vascular-massa miocárdica é desfavorável.

Embora a necrose subendocárdica seja há muito conhecida como complicação de perfusão coronária cirúrgica, o conceito de lesäo focal miocárdica é ressaltado em estudo recente de ALDEA et al (22), demonstrando que o padrăo de distribuiçāo seg. mentar do fluxo coronário varia, mesmo na ausência de obstruções arteriais, o que renova o interesse pelo intrigante fenômeno de derivação de fluxo coronário demonstrado em 1979 (23).

A sistematização da cardioplegia retrógrada, foi amplamente pesquisada $\theta$ divulgada por BUCKBERG (24) e DRINKWATER et al. (11), empregando cânula com balăo auto-inflável no seio coronário. MENASCHÉ et al. (10) descreveram 1500 cirurgias, com bons resultados, empregando apenas a perfusăo cardioplégica retrógrada, por canulação sob visăo direta do seio coronário, como método de proteção miocárdica.

FABIANi et al. (25) utilizaram apenas a periusāo retrógrada total, atrial, como proteçāo miocárdica em grande número de pacientes, com bons resultados.

A cardioplegia retrógrada total, atrial, embora eficaz, estende muito a cirurgia exigindo volume e tempo de infusão muito maiores que nas demais.
O entusiasmo inicial com emprego de cardioplegia retrógrada seletiva ( $\mathrm{SCo}$ ), isoladamente tem diminuído, face às investigações que demonstram proteção heterogênea do mícárdio com seu emprego $(13,22,26)$, principalmente ao nível de VD, enquanto cresce o uso combinado das formas anterógrada $\theta$ retrógrada seletiva $(11,12)$.

Na presente investigação, pode-se confirmar os resultados das pesquisas acima, demonstrando melhor perfusâo miocárdica no ventrículo esquerdo com as técnicas anterógrada e retrógrada seletiva, contrastando com melhor proteçảo das câmaras direitas na cardioplegia retrógrada total.

Confirmou-se também que a técnica retrógrada total exigiu volume e tempo de perfusāo significantemente maiores que nas demais técnicas, o que se pode considerar como efeito adverso, tendo em vista que, durante todo o período de injeção, as câmaras direitas ficam muito distendidas, prejudicando a exposição cirúrgica.

O emprego de cardioplegia retrógrada sequeencial na variante SCo-AD possibilitou proteçăo cardiaca total superior, ao nível de câmaras direitas e região do nó sinoatrial, à obtida com a técnica anterógrada.

A cardioplegia retrógrada seqũencial tipo SCoVD, passivel de utilização mesmo nas intervenções que exijam abertura do átrio direito, mostrou proteçăo estatísticamente semelhante à anterior, quanto ao VE e VD, ficando menos protegidos $A D$ e regiāo sinusal.

Com base nos resultados da presente investigaçăo, pode-se concluir que a técnica de cardioplegia retrógrada seqũencial ora proposta é significantemente melhor para proteçāo miocárdica em cirurgia do que as técnicas usuais de perfusăo retrógrada seletiva pelo seio coronário, ou total pelo átrio direito, empregadas isoladamente, quando comparadas com os resultados da cardioplegia anterógrada. 
Gomes O M, Pitchon M, Brum J M G, Gomes E S, Pádua Filho W C, Freitas A E A, Faraj M, Lima W S. Sequencial retrograde cardioplegy. Rev Bras Cir Cardiovasc 1996;11 (3): 208-15.

ABSTRACT: The distribution pattern of a cold $\left(3-4^{\circ} \mathrm{C}\right)$, crystalloid cardioplegic solution (CS) in the myocardium was studied in 15 mongrel dogs, with $10-15 \mathrm{~kg}$ of body weight. After anesthesia and median sternal thoracotomy. The pericardium was opened and extracorporeal circulation established. The following routes were employed for cardioplegic perfusion:1) Antegrade - through ascending aortic canullation bellow the aortic occlusion clamp; 2 ) Selective retrograde - through coronary synus (Co. $\mathrm{S}-25 \mathrm{mmHg}$ ) using a selfinflating ballooned cannula; 3 ) Total retrograde, (Co.S $-40 \mathrm{mmHg}$ ) - through a cannula inserted in the right atrium (RA); 4) Sequencial retrograde, Co.S-RA - with the CS flowing first through the coronary synus lowering the interventricular septal temperature to $16^{\circ} \mathrm{C}$ and after through the RA cannula as in the total retrograde technique with the pulmonary artery occluded and;5) Sequencial retrograde, Co.S-RV - the RV chamber being directly cannulated through the tricuspid valve and perfused, instead of the RA in the latter technique. The temperature variation of the myocardium in the left ventricule (LV), RV, RA and sinus node region (SN) was controlled employing an Omega needle termistor and thermometer. With the antegrade technique ( $70 \mathrm{mmHg}$ pressure) the most uniform myocardial cooling, the lowest CS volume and perfusion time duration was observed, followed in excelence by the Co.S-RA Sequencial retrograde technique and the Co.S. RV sequencial technique. The present data indicate that sequencial retrograde cardioplegic perfusion techinique is significantly better than the usual Co.S or RA total retrograde technique alone for myocardial protection when compared with the aortic root antegrade perfusion technique.

DESCRIPTORS: Heart arrest, induced, methods. Myocardium, metabolism. Cardioplegy. Myocardial protection, cardioplegy.

\section{REFERÊNCIAS BIBLIOGRÁFICAS}

1 Melrose D G, Dreyr B, Bertoll H H. Elective cardiac arrest. Lancet 1955; 2: 21-2.

2 Reidemeister J C, Gehl H, Spieckman P G, Oreland L E - Untersuchung der Kardioplegie durch extrazellularen Natrium und Calciumentzung und Novocanngable in Uberlebensversuch am hund. Langenbecks Arch Lin Chir 1965; 313: 1043-81.

3 Bretschneider H S, Ubner G, Knoll D, Lohr B, Nordbeck $H$, Spieckmann P E - Myocardial resistance and tolerance to ischemia: physiological and biochemical basis. J Cardiovasc Surg 1975; 16: 241-60.

4 Kirsch U, Head L R, Nogueira C - Direct coronary artery perfusion for aortic valvular surgery. JAMA 1958; 168: $1767-73$.

5 Kay E B, Head L R, Nogueira C - Direct coronary artery perfusion for aortic valvar surgery. JAMA 1958; $168: 1774-76$.

6 Lillehei C W, De Wall R A, Got V L, Varco R L. The direct vision correction of calcific aortic stenosis by means of a pump-oxygebtor and retrograde coronary perfusion. Dis Chest 1956; 30: 123-33.

7 Morais D J - Perfusāo coronária retrógrada. Apud: Jazbik A P. Nova técnica de perfusaáo retrógrada do miocárdio: estudo clínico e experimental. [Tese] Rio de Janeiro: Faculdade de Medicina da Universidade Federal do Rio de Janeiro, 1970. clinico e experimental [Tese] Rio de Janeiro: Faculdade de Medicina da Universidade Federal do Rio de Janeiro, 1970.

9 Menasché $\mathrm{P}$, Kural S, Fauchet M - Retrograde coronary sinus perfusion: a safe alternative for ensuring cardioplegic delivery in aortic valve surgery. Ann Thorac Surg 1982; 34: 647-58.

10 Menasché P, Subayi J B, Piwinica A - Retrograde coronary sinus cardioplegy for aortic valve operations: a clinical report on 500 patients. Ann Thorac Surg 1990; 49: 556-64.

11 Drinkwater D C, Lakes H, Buckberg G D - A new simplified method of optimizing cardioplegic delivery without heart isolation. J Thorac Cardiovasc Surg 1990; 100: 56-64.

12 Partington M T, Acar C, Buckberg G D, Julia P L . Studies of retrograde cardioplegia. II: Advantages of antegrade/retrograde cardioplegia to optimize distribution and jeopardize myocardium. $J$ Thorac Cardiovasc Surg 1989; 97: 613-22.

13 Stirling M C, McClanhan T B, Schott R J, Lynch M J, Bolling S F, Kirsch M M - Distribution of cardioplegic solution infused antegradely and retrogradely in normal canine hearts. I Thorac Cardiovasc Surg 1989; 98: 1066-76.

14 Roe B B \& Kelley P B - Perfusion through the ascending aorta: experience with 410 cases. Ann Thorac Surg $1969 ; 7: 238-43$.

15 Morais D J, Jazbik W, Jazbik A P, De Biase H, Brito J 

seqüencial. Rev Bras Cir Cardiovasc 1996; 11 (3): 208-15.

Jazbik A P, Jazbik W, Meier M A et al. - Proteçăo do miocárdio, com nutriçăo coronária retrógrada. Arq Bras Cardiol 1969; 22: 83-4.

17 Braile D M, Bilaqui $A$, Anacleto $\mathrm{J} \mathrm{C}$ et al. - Proteçāo miocárdica por cardioplegia. Arq Bras Cardiol 1979; 33 (Supl. 1): 199-210.

18 Shumway N E, Lower R R, Stoffer R C - Selective hypothermia of the heart in anoxic cardiac arrest. Surg Gynec Obstet 1959; 109: 750-54.

19 Hufnagel C A, Conrad P W, Schanno J, Pifarri R Profound cardiac hypothermia. Ann Surg 1961; 153: 790-6.

Jatene A D - Hipotermia seletiva do miocárdio. Rev Assoc Med Bras 1963; 9: 114-21.

21 Fabiani J N, Deloche A, Swanson J, Carpentier A Retrograde cardioplegia through the right atrium. Ann Thorac Surg 1986; 41: 101-2.
22 Aldea G S, Austin Jr. R E, Flynn A E, Coggins D L, Husseini W, Hoffman J I E - Heterogeneous delivery of cardioplegic solution in the absence of coronary artery disease. J Thorac Cardiovasc Surg 1980; 99: $345-50$.

23 Gomes O M, Barros-Moraes N L T, Fiorelli A I et al. Fenómenos de derivaçăo do fluxo coronário: primeira demonstraçăo experimental. Arq Bras Cardiol 1979; 32: 141-5.

24 Buckberg G D - Antegrade cardioplegia, retrograde cardioplegia, or both? Ann Thorac Cardiovasc Surg 1988; 45: 589-90.

25 Fabiani J N, Swanson J, Deloche A, Carpentier A - Right atrial cardioplegia. In: Roberts A J, ed. Myocardial protection in cardiac surgery. New York: Marcel Dekker, 1987.

26 Dembitsk W P - Discussion. In: Drinkwater D C, Lakes $H$, Buckberg G D. New simplified method of optimizing cardioplegic delivery without right heart isolation: antegrade/retrograde cardioplegia. J Thorac Cardiovasc Surg 1990; 100: 56-64. 